\title{
Current nursing practices for managing children with burn injuries
}

Ghada Elbana, Hanafi Zoorob, Saad Shaaban and Wafaa Hamama

Department of Chemistry, Faculty of Science, Mansoura University, Egypt

\section{III}

Background: Triazines represent a promising scaffold for cancer treatment due to their similarity to the biologically active purine and pyrimidine systems. Besides, triazines and annulated compounds exist in the skeleton of numerous natural and synthetic chemotherapeutic agents such as Azanucleosides (6-azacytosine and 6-azauracil). Also, triazine derivatives proved to display antiviral, anti-inflammatory, antimicrobial and antifungal activities. Moreover, it was found that triazine derivatives display significant wide spectrum anticancer activities against lymphoblastic lung adenocarcinoma A549, leukemia CEM and myeloid leukemia K562 cancer cell lines. Aim: Test the antimicrobial and antitumor activities of nitrogenous precursors in a fused or binary heterocyclic system. Materials and Methods: Newly series of triazine-based thiadiazol, thiadiazine, thiadiazepine, pyrazole tetrazepino[b]indol-one, triazolotriazine, tetrazolotriazine, triazinotetrazine, and triazinotetrazepinoindole moieties were synthesized, spectrally discussed and mechanistically discussed. On the other hand, the synthesized compounds were evaluated against different types of biological bioassays (Antitumor, Antioxidant and Antimicrobial). Also, compounds 33c and 37 were evaluated against three human cancer cell lines (HepG-2, HCT-116, and MCF-7) for their in-vitro anticancer activities. Results: The results declared that all compounds had a potent anticancer activity with IC50 values ranging between 10.8 and $23.4 \mu \mathrm{M}$. Conclusion: Th synthesized nitrogenous precursors in a fused or binary heterocyclic system expressed antimicrobial and antitumor activities.

Keywords: Anti-tumor; Anti-oxidant; 1,2,4-Triazine

Editor-in-Chief: Prof. M.L. Salem, PhD - Article DOI: 10.21608/JCBR.2021.60293.1148 